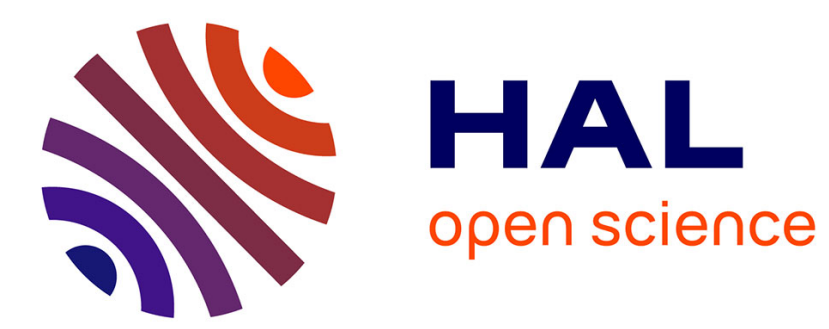

\title{
A ville plus grande, travail plus qualifié
}

Thérèse Saint-Julien, Denise Pumain

\section{To cite this version:}

Thérèse Saint-Julien, Denise Pumain. A ville plus grande, travail plus qualifié. Les Annales de la Recherche Urbaine, 1986, 29, pp.105-118. halshs-01565271

\section{HAL Id: halshs-01565271 https://shs.hal.science/halshs-01565271}

Submitted on 27 Oct 2017

HAL is a multi-disciplinary open access archive for the deposit and dissemination of scientific research documents, whether they are published or not. The documents may come from teaching and research institutions in France or abroad, or from public or private research centers.
L'archive ouverte pluridisciplinaire HAL, est destinée au dépôt et à la diffusion de documents scientifiques de niveau recherche, publiés ou non, émanant des établissements d'enseignement et de recherche français ou étrangers, des laboratoires publics ou privés. 


\title{
A ville plus grande travail plus qualifié
}

\author{
Thérèse Saint-Julien, Denise Pumain
}

Dans le processus contemporain d'urbanisation, les années 1975-1980 sont un moment important. En France, la brutalité du ralentissement de la croissance de la population urbaine a surpris. Quoique inégalement, les taux d'augmentation de la population des villes ${ }^{1}$ se sont effondrés après 1975. Le fort ralentissement de la croissance des emplois industriels, entre 1970 et 1975, annonçait le déclin enregistré par la suite, déclin que la croissance des emplois de service n'a que partiellement compensé. Les années récentes sont enfin marquées par l'inadéquation croissante, quantitative et qualitative, entre emplois offerts et emplois demandés et par le renforcement continu et rapide d'une nouvelle catégorie de population, celle des actifs à la recherche d'un emploi. L'état de chômeur introduit dans les villes une nouvelle catégorie d'«actifs» dont le poids n'a cessé de croître depuis 1975. Le chômage est désormais une composante importante des profils d'emploi urbain. Bien qu'il soit impossible, en l'état actuel de la saisie des données sur l'emploi, d'en connaître l'importance réelle à cet échelon géographique, on sait qu'il est un phénomène désormais grave, avec lequel toutes les villes doivent compter.

Dans ie contexte général de crise, de mutation technico-économique, en rupture avec celui des décennies précédentes, nous caractériserons l'évolution de la division interurbaine du travail ${ }^{2}$. En effet, les transformations qualitatives de cette division du travail sont symptomatiques des réaménagements que subit aujourd'hui le réseau urbain national, et par lui l'espace français qu'il structure. L'importance des problèmes quantitatifs posés aujourd'hui par l'évolution de l'emploi a tendance à faire passer au second plan les transformations qualitatives essentielles, mais difficiles à définir. En période de transition, les cartes sont un peu brouillées. Dans la dynamique urbaine actuelle, l'ancien et le nouveau interfèrent : comment déceler ce qui est dernier souffle d'une phase d'expansion révolue ou au contraire signe d'un nouvel ordre de choses?

En dépit de ce que laisserait supposer une approche superficielle, le concept de qualification du travail est difficile à cerner. Iribarne et Virville [19*] ont bien montré que la qualification se définit à l'intersection des systèmes productif et éducatif : «Elle est constituée par un potentiel qui est, entre autres, acquis à travers l'appareil éducatif et qui se manifeste lors de sa mise en œuvre dans le processus de travail.» Elle est bien une réalisation à la

1. Nous employons indifféremment les expressions «ville», "agglomération» ou "unité urbaine»: dans tous les cas, il s'agit des unités urbaines (définition INSEE 1975).

2. Cette étude a fait l'objet d'une convention de recherche entre I'UA Economie/Espace/Environnement, la Jeune Equipe CNRS PARIS et le ministère de l'Urbanisme et du Logement : la Division interurbaine du travail en France, la crise et l'évolution de la qualification du travail dans les villes.

* Les chiffres entre crochets renvoient à la bibliographie infra, p. 117. 
jonction, parfois contradictoire, très souvent conflictuelle, de la demande de travail émanant des individus et de l'offre proposée par le système productif. En période d'accentuation de la division spatiale du travail, la qualification de celui-ci est progressivement devenue une médiation de premier plan dans la relation dialectique qui associe devenir économique et devenir social des groupes. Elle est par là même une expression centrale des restructurations socio-économiques qui se manifestent dans et entre les territoires de ces groupes.

Entre les villes, les disparités de la qualification du travail restent indissociables de l'inégale importance locale des tensions et des «drames » de l'emploi, mais aussi de l'inégale aptitude des unités urbaines à créer, à s'adapter, en un mot à répondre à la crise. A la fin de la dernière décennie, l'attention a été attirée $[1,2,4 ; 22$; 27] sur le nouveau rôle joué par le travail dans la structuration des réseaux urbains. La diversité interurbaine tendait, moins que par le passé, à reposer sur la nature des activités présentes dans

3. Les agglomérations françaises qui avaient au moins 10000 habitants en 1975 (soit 409) ont été notre champ d'observation. Nous avons saisi ces changements à partir de l'enquête annuelle "Structure des emplois" du ministère du Travail. Certes, cette source a ses limites : l'enquête ne concerne que l'emploi salarié des établissements occupant au moins dix personnes. Son taux de couverture varie sensiblement d'une activité à l'autre (de $85 \%$ et plus dans le secteur secondaire à $45 \%$ environ dans le secteur tertiaire). Mais elle a l'immense intérêt de répertorier ces emplois selon une nomenclature qui décrit assez bien les niveaux de qualification du travail (ingénieurs et cadres, techniciens, agents de maîtrise, emplois d'exécution qualifiés et emplois d'exécution non qualifiés) parmi les emplois de production d'une part, les emplois de service d'autre part. (Une analyse critique très détaillée de la source a été réalisée dans l'étude ci-dessus mentionnée, p. 105, note 2). Nous disposons donc là d'un outil privilégié pour mesurer les effets des stratégies spatiales des agents économiques et la redistribution des fonctions dans le réseau urbain. Cet outil a permis aussi d'apprécier l'ampleur et l'évolution des disparités spatiales en termes de savoir, de pouvoir et de richesse associés aux inégales concentrations de chaque type d'emploi.

Partant de la situation de 1975 , nous aurions aimé prolonger cette période d'observation jusqu'à une date aussi proche que possible de l'année 1984. Les lenteurs de la saisie et de la mise à disposition de données relatives à l'ensemble des unités urbaines de plus de 10000 habitants nous ont imposé de nous satisfaire de l'année 1980 comme année ultime de référence. Nous gardons présent à l'esprit que cette date est tout au plus un repère commode pour saisir les grandes tendances du changement qui se manifeste aujourd'hui dans le partage interurbain du travail et dont les nouvelles modalités ne sont pas encore bien fixées. les villes et davantage sur le niveau de qualification du travail requis par ces activités. Les vérifications entreprises, sur l'ensemble du système urbain et pour la période 1954-1975 [27, $28 ; 29 ; 26]$, ont montré qu'un très léger infléchissement des transformations en cours s'était progressivement manifesté dans ce sens. On peut difficilement imaginer que, depuis 1975 l'amplitude de ces disparités se soit amenuisée.

Le début des années soixante-dix avait livré les signes annonciateurs de ce qu'on pourra bien un jour appeler la troisième révolution industrielle. Les domaines innovants des prochaines décennies commencent à pouvoir être identifiés, les activités motrices ne sont plus les mêmes, les frontières entre activités de production et de services sont beaucoup moins nettes que par le passé et cette évolution, à bien des égards spectaculaire, s'effectue sur fond d'un profond et massif renouvellement des modes de production et de circulation de l'information.

On pourrait penser que tout cela a bouleversé de fond en comble les tendances de l'évolution antérieure de la division qualitative interurbaine du travail. Or, il n'en est rien : au contraire, certains de ses traits ont été légèrement accentués, au bénéfice des grandes villes surtout, dans la mesure où l'évolution récente doit être considérée comme le stade primaire d'un vaste processus de diffusion spatiale des innovations, porteuses du changement. On sait en effet que, pour un territoire national donné, et à ce stade primaire du processus de diffusion, les probabilités que les plus grands centres urbains soient les premiers atteints sont grandes [30]. On sait aussi que l'apparition en un lieu d'une innovation, déterminante pour que se réalise l'apparition d'un foyer initial d'émission - et que s'engage à partir de lui un processus de développement ne peut s'effectuer sans qu'existent ou que se constituent en ce lieu des potentialités de travail requérant un haut niveau de savoir-faire.

Notre étude répond à trois séries de questions. La première a trait à l'éventuel renforcement des discriminations que la qualification du travail introduisait déjà en 1975 entre les unités urbaines françaises ${ }^{3}$ et à l'examen de tout ce qui peut être interprété comme une modification des fondements de ces discriminations depuis dix ans. 
Il existe de réelles difficultés, dans cette période de transition, à démêler, dans les changements observés, ce qui est expression de la fin d'une étape et ce qui, au contraire, est porteur d'avenir et prépare le réseau urbain de demain. Pour dépasser cette contradiction et tourner délibérément notre réflexion vers le futur de ce réseau, nous isolons un certain nombre d'activités - industrielles surtout, mais aussi tertiaires - que l'on peut considérer comme des activités de pointe, susceptibles d'être plus sensibles que d'autres aux localisations et aux ségrégations géographiques du futur. Nous cherchons à déterminer si, entre 1975 et
1980, leur comportement spatial s'était démarqué de l'ensemble, annonçant ainsi des transformations prévisibles à terme.

Nous examinons enfin suivant quels mécanismes une ville particulière a été en mesure d'assimiler, d'infléchir, de corriger les processus généraux de changement. L'observation locale de l'évolution d'un bassin urbain d'emploi $a$ priori ni mieux ni plus mal loti que l'ensemble, celui de l'agglomération de Blois, nous a semblé le meilleur moyen pour répondre à l'interrogation portant sur la nature particulière des mécanismes locaux de changement en matière de qualification du travail.

\section{Plus les villes sont grandes, plus l'emploi y est qualifié}

Dès 1975, les niveaux de qualification du travail salarié créent dans le système des villes françaises des spécialisations marquées. Ces spécialisations sont d'autant plus accusées et d'autant plus exclusives les unes des autres qu'elles sont le fait de niveaux de qualification éloignés sur l'échelle hiérarchique du travail (tableau 1). Dans une agglomération spécialisée

1. Grands types urbains de qualification du travail en $1975^{\prime}$

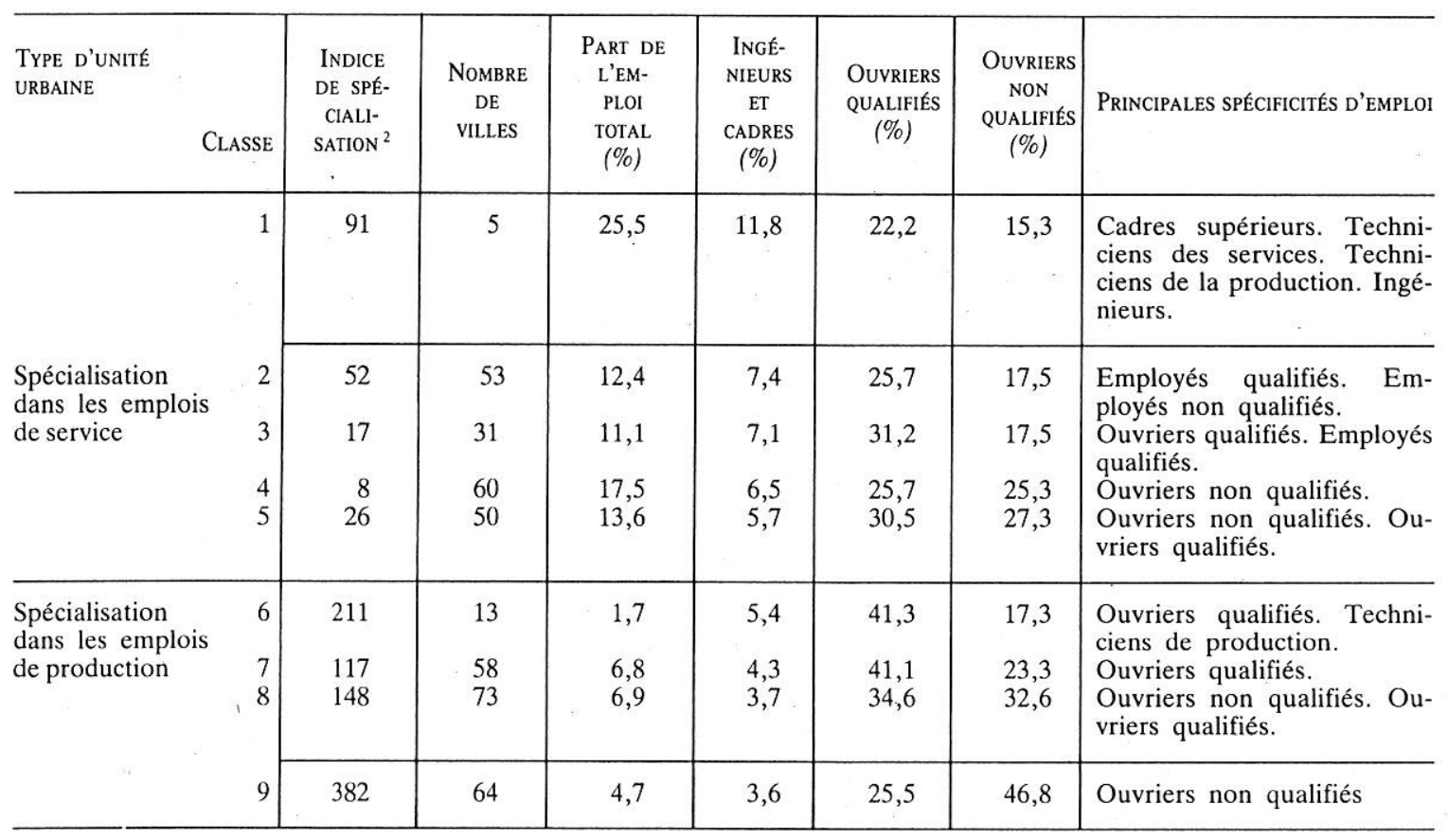

1. Classification ascendante hiérarchique (distance du $X^{2}$ ) effectuée sur un tableau décrivant les effectifs salariés de chaque unité urbaine suivant cinq niveaux de qualification pour les emplois de production d'une part, pour les emplois de service d'autre part.

2. Indice de spécialisation : distance $\left(\mathrm{X}^{2}\right)$ du centre de gravité de la classe au centre de gravité de l'ensemble. Si l'indice est égal à 0 , l'emploi de la ville n'est pas spécialisé ; plus l'indice croît, plus l'emploi de la ville est spécialisé. 
dans les emplois de production, il ne peut y avoir surreprésentation conjointe des ouvriers, qualifiés ou pas, et des cadres et ingénieurs. Au contraire, la catégorie des ouvriers sans qualification d'une part, celle des cadres et ingénieurs de la production d'autre part sont chacune à l'origine de spécialisations du travail urbain très antinomiques et concernent des sous-ensembles de villes différents. La division interurbaine du travail passe donc grandement par les clivages hiérarchiques qu'expriment les différents niveaux de qualification.

La force des oppositions géographiques ainsi créées dans le réseau urbain, donc l'importance des interdépendances qu'elles y engendrent, sont beaucoup plus grandes à l'intérieur des emplois de production qu'à l'intérieur des emplois de service. La division spatiale du travail, réalisée sur la base d'une relative ségrégation géographique des niveaux de qualification, est en 1975 et reste en 1980 - beaucoup plus avancée pour les emplois de production que pour les emplois de service.

Au-delà de ce principe majeur de la différenciation interurbaine du travail, les autres caractéristiques de spécialisation sont mineures. Les inégalités de qualification du travail ouvrier en sont le fondement essentiel. Les emplois tertiaires d'exécution apparaissent à ce niveau beaucoup moins discriminants.

Les concentrations géographiques des types d'emploi dans les villes, leurs associations et exclusions dans le réseau sont, pour l'emploi féminin, sensiblement différentes de ce qu'elles sont pour l'ensemble des salariés. On observe une dissociation beaucoup plus accusée entre une majorité de villes d'ouvrières et celles, beaucoup moins nombreuses, où ingénieurs et techniciennes sont surreprésentées. Dans la définition des spécialisations, la hiérarchie des emplois de service est un peu bouleversée, la catégorie des agents de maîtrise est beaucoup plus inégalement répartie entre les villes que celle des cadres supérieurs. Surtout, une différence essentielle entre la division interurbaine du travail féminin et celle de l'ensemble - et donc celle du travail masculin - réside en une distinction beaucoup plus tranchée, parmi les villes ouvrières, entre celles où dominent les emplois d'ouvrières qualifiées et celles où les ouvrières sont en majorité non qualifiées. $\mathrm{Au}$ contraire, si l'on considère le seul travail mascu- lin, cette opposition demeure beaucoup moins accusée.

La distribution géographique des grands types de profils urbains d'emplois, définis d'après la hiérarchie des qualifications, épouse à certains égards l'organisation hiérarchique du réseau des villes. Deux régularités essentielles apparaissent : la première est que, lorsqu'on s'élève dans la hiérarchie de taille des agglomérations, la tertiarisation des emplois d'exécution a tendance à augmenter en moyenne. Tout se passe comme si, à des emplois d'ouvriers, se substituaient des emplois d'employés. La seconde régularité mise en évidence est une élévation du niveau de qualification du travail avec la taille des villes. Pour les emplois de production, cette élévation de la qualification est due conjointement à l'augmentation du poids relatif des emplois de cadres et techniciens et à celle du degré de qualification des emplois ouvriers. Pour les emplois de service, cette élévation du niveau de qualification avec la taille des unités urbaines repose exclusivement sur l'accroissement du poids relatif des cadres et techniciens. Paris excepté, le degré de qualification des salariés, à l'intérieur de la catégorie des employés, semble, en moyenne, à peu près indépendant de la taille des villes.

A cet égard, une remarque importante doit être faite. Quand on isole, de l'ensemble des emplois, ceux de l'industrie (bâtiment et travaux publics exclus), on constate que le degré moyen de qualification des employés de ce secteur s'élève régulièrement avec la taille des agglomérations, au-delà du seuil de 20000 habitants. On peut considérer que cette spécificité de la distribution interurbaine des emplois industriels de service est l'expression d'interactions plus grandes entre les exigences de ce tertiaire peu banal en matière de localisation et les potentialités différentielles que recèlent les niveaux de la hiérarchie urbaine.

Enfin, par rapport à l'ensemble, la division du travail féminin entre les villes de tailles différentes s'individualise sur un point: le degré moyen de qualification des emplois de production est à peu près indépendant de la taille des villes; cette indépendance est particulièrement sensible pour les emplois d'ouvrières.

Au total, la variété des situations urbaines à l'intérieur d'une même classe de taille n'est certes pas négligeable, mais la diversité y est en moyenne moins grande qu'elle ne l'est dans 
l'ensemble des agglomérations. On constate d'ailleurs que la distribution des villes entre les différents types de profils de qualification du travail urbain est assez largement reliée à celle de leur taille. Plus une ville est petite, plus grande est la probabilité que son profil d'emploi soit marqué par une spécialisation dans l'activité de production, dans les emplois ouvriers faiblement qualifiés surtout. Au contraire, au-delà du seuil de taille de 50000 habitants, la spécialisation se fait plutôt dans les emplois de services, avec un taux d'encadrement de plus en plus élevé : ainsi, toutes les capitales de région de programme appartenaient dès 1975 à des classes caractérisées par une surreprésentation de ces catégories très qualifiées.

L'apparition de ces spécialisations et les ségrégations interurbaines qu'elles engendrent ne sont pas non plus totalement indépendantes de l'appartenance des agglomérations à un réseau urbain régional particulier. La spécificité plus ou moins industrielle ou tertiaire de l'activité régionale, la position plus ou moins centrale de l'espace régional dans le réseau et, plus secondairement, la forme régionale de ce réseau interfèrent dans la définition de la nature et des niveaux de spécialisation des profils urbains d'emploi. On trouve les villes du Nord et de l'Est plutôt définies par des surreprésentations ouvrières et, à l'opposé, les agglomérations du Sud-Est et surtout de la région parisienne davantage concernées par des surreprésentations dans les catégories de cadres et de techniciens de la production et des services, les régions du Bassin parisien, du Centre-Est et de l'Ouest étant en position intermédiaire.

\section{L'inégalité des qualifications entre villes s'accrô̂t au bénéfice des plus grandes}

Les changements intervenus dans la distribution interurbaine des emplois au cours des cinq dernières années n'ont pas été de nature à bouleverser la structure de 1975, et les images qu'on pourrait donner de celle de 1980 en sont très proches. Il en est ainsi parce que les transformations communes à toutes les villes ont été les plus importantes. Deux tendances principales les caractérisent.

La première tendance est la substitution relative des emplois de service aux emplois de production. Ce processus de tertiarisation est sensible, que l'on considère l'évolution d'ensemble, celle des profils des différentes classes de taille ou celle des différentes régions. Il s'agit là du prolongement d'une tendance antérieure, relativement ancienne, et qui touche, entre 1975 et 1980, la quasi-totalité des agglomérations (une vingtaine seulement font exception).

La seconde tendance correspond à un relèvement des niveaux moyens de la qualification du travail dans les villes. Elle se traduit par un renforcement du poids relatif des catégories d'ingénieurs, de cadres et de techniciens de la production et des services et par un déclin sensible de l'importance relative des ouvriers non qualifiés, plus accentué que ne l'a été celui de l'ensemble de l'emploi ouvrier. Cette évolution vers une amélioration de la qualification relative des emplois ouvriers ne concerne cependant que les emplois masculins. En effet, si, pour les femmes, le taux de technicité ${ }^{4}$ des emplois de production se relève très rapidement entre 1975 et 1980 , le niveau moyen de qualification des ouvrières demeure stable et à peu près identique, quelle que soit la taille des villes.

A. Valeyre [34] avait évoqué la possibilité que la crise mette un terme à la déqualification relative du travail ouvrier. Nous retrouvons, à l'échelle de ce vaste ensemble de villes, le signe que de telles évolutions sont en cours pour le travail masculin. Elles sont l'expression de la fin d'un cycle d'expansion de l'emploi industriel qui a reposé sur la croissance très rapide du nombre des postes de travail n'exigeant qu'une maind'œuvre banale. Le relais n'est aujourd'hui pris, en la matière, par aucune activité nouvelle et partout se manifeste un tassement global de l'emploi industriel d'exécution ne nécessitant

4. On appelle taux de technicité, la proportion des emplois d'ingénieurs, cadres et techniciens dans l'ensemble des emplois de production ou de service, ou du total des salariés. 
I. Les grands types de qualification du travail en 1975

1. Unités urbaines plutôt spécialisées, dans les emplois de production

\section{Avec une surreprésentation des}

techniciens de la production et des ouvriers qualifiés ouvriers qualifiés

ouvriers qualifiés et non qualifiés

ouvriers non qualifiés

\section{Unités urbaines plutôt spécialisées} dans les emplois de service

\section{Avec une surreprésentation des}

cadres et techniciens de la production et des services employés qualifiés et non qualifiés ouvriers et employés qualifiés ouvriers non qualifiés

ouvriers qualifiés et non qualifiés

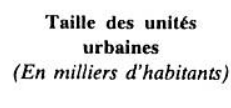

1. Plus de 2000 . 2. $200-2000 .-3.100$ - 200. - 4. 20 - 50 .

\section{Taille des unités urbaines \\ (En milliers d'habitants)}

$\begin{array}{lllll}1 & 2 & 3 & 4 & 5\end{array}$

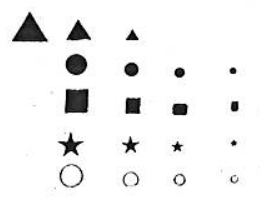

1. Plus de 2000 . 2. $200-2000 .-3.100$ $-200 .-4.20-50$.

\section{Taille des unites urbaines (En milliers d'habitants)}

II. Les grands types de changements différentiels
des profils urbains d'emploi entre 1975 et 1980

Accélération des processus de tertiarisation avec amélioration de la technicité des services et de la qualification des employés

amélioration de la technicité de la production et de la qualification des employés

amélioration de la technicité des services avec détérioration de la qualification des employés

Freins à la tertiarisation avec

amélioation de la technicité de la production

amélioration de la technicité de la production et détérioration de la qualification des employés

amélioration de la qualification ouvrière

détérioration de la qualification ouvrière

forte détérioration de la qualification ouvrière $\begin{array}{lllll}1 & 2 & 3 & 4 & 5\end{array}$ is it it (1)

O 00

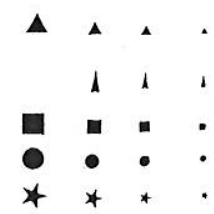

1. Plus de 2000 . 1. Plus de $2000 .-$
2. $200-2000,-3.100$ $-200 .-4.50 \cdot 100$. 5. $20-50$.
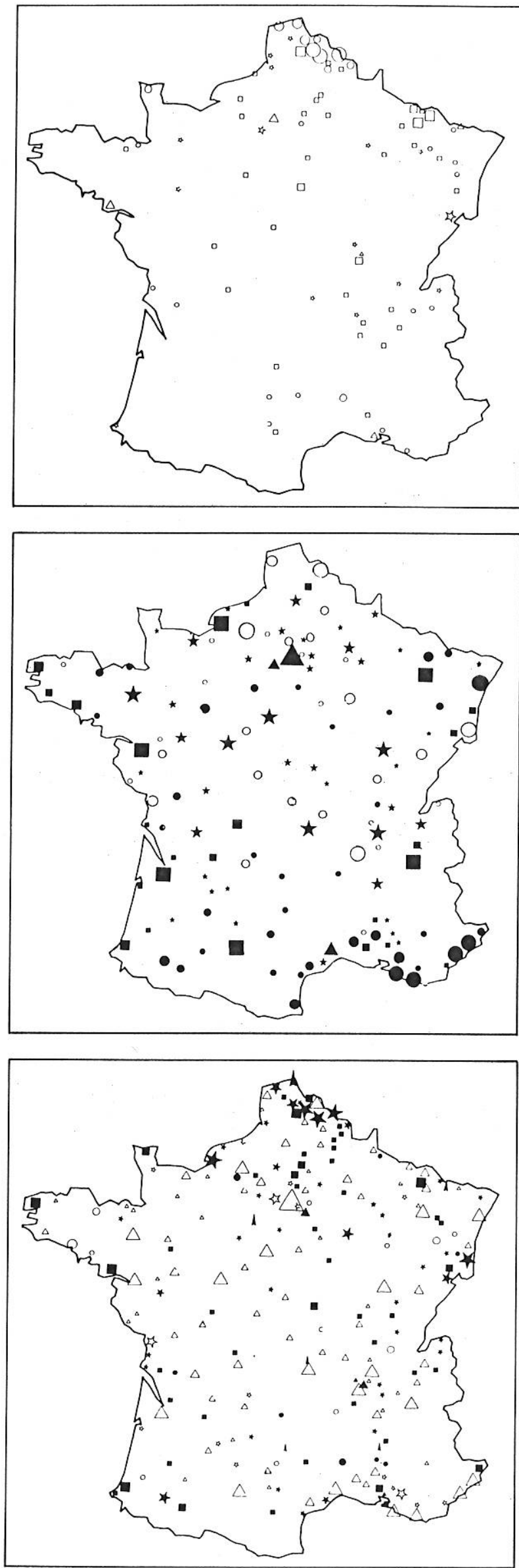
aucune qualification particulière. L'heure est plus au recentrage et à l'inventaire de nouvelles voies possibles, et les compétences requises ne sont plus les mêmes. A l'intérieur des emplois d'exécution des services, on n'observe pas de transferts équivalents. Les proportions relatives d'employés qualifiés et non qualifiés ont crû à peu près au même rythme entre 1975 et 1980 .

Ces tendances ont eu une telle généralité que les évolutions des profils d'emploi des agglomérations ont été le plus souvent très voisines. De ce fait, les principes suivant lesquels s'organise, entre les villes, la division du travail salarié ont été peu retouchés par ces évolutions. En 1980, comme en 1975, la hiérarchie des qualifications fonde pour une large part la diversité des spécialisations des profils urbains d'emplois.

Des transformations propres au profil de qualification de chaque ville peuvent apparaitre, en effet, si les changements intervenus ne sont pas de même nature que dans les autres villes. Elles peuvent aussi résulter des différentes vitesses suivant lesquelles les agglomérations ont enregistré les changements les plus généraux. C'est surtout pour cette dernière raison que les transformations récentes des profils urbains d'emploi ont été génératrices de certaines retouches dans les positions relatives des agglomérations. Nous avons isolé et identifié la nature et l'ampleur de ces changements différentiels et observé que, dans l'ensemble, ils ne sont pas indépendants les uns des autres.

En dépit d'une relative diversité des évolutions des villes, on observe une certaine concomitance entre les modifications enregistrées pour des catégories d'emploi déjà associées dans la structure d'ensemble de l'organisation interurbaine du travail en 1975. Les changements les plus importants et les plus fréquents consistent en deux mouvements opposés. L'un traduit le renforcement relatif des emplois d'exécution de la production. Il est le fait de villes dont l'évolution est allée soit à l'encontre du mouvement d'ensemble de tertiarisation, soit dans le sens de ce mouvement, mais plus lentement que l'ensemble des villes. En fait, c'est ce dernier scénario qui a prévalu. Il s'est accompagné, dans un peı moins des deux tiers des cas, d'un renforcement relatif, plus rapide qu'ailleurs, de la qualification ouvrière. Le second mouvement correspond à l'accélération des substitutions des emplois de service aux emplois de production, avec accentuation relative plus rapide qu'ailleurs du niveau de technicité du travail.

La portée de ces changements différentiels n'est pas négligeable, car leur apparition dans le réseau n'est pas aléatoire. Elle conduit, dans au moins deux cas sur trois, à accentuer l'écart initial au profil moyen. En effet, l'apparition de ces changements est à la fois fonction du profil d'emploi de 1975 et de la position hiérarchique de la ville dans le réseau. Parmi les villes à structure initiale caractérisée par une spécialisation dans les services, ce sont plutôt les grandes (la plupart d'entre elles appartiennent à ce groupe) qui renforcent plus rapidement que les autres la tertiarisation et la qualification de leur profil. Les plus petites ont au contraire tendance à diversifier leur structure d'emploi par des substitutions au profit d'emplois de production souvent peu qualifiés. Les villes initialement très spécialisées dans les emplois de production évoluent de manières très diverses sans que la taille paraisse introduire une discrimination particulière. Elles ont peu de chances de connaître un processus d'accélération de la tertiarisation de leurs emplois, mais ne sont pas condamnées à des évolutions similaires de la spécificité de leurs emplois de production.

On constate donc l'émergence d'un modèle «grande ville» des profils d'emploi, émergence dont nous avions pu déceler l'apparition au cours de la période intercensitaire 1968-1975 [26].

Nous avons en vain cherché à voir s'il existait quelque relation entre ces évolutions spécifiques, plutôt favorables aux grandes villes, et d'autres caractéristiques de la dynamique urbaine actuelle (évolution démographique, évolution de la concentration technique des emplois, par exemple). En identifiant la nature et l'ampleur des transformations spécifiques des profils d'emploi des villes entre 1975 et 1980 , nous saisissons d'abord l'expression des inégalités de leurs positions relatives par rapport aux foyers d'innovation à partir desquels s'orchestrent aujourd'hui les mutations socio-économiques. 


\section{Les activités de pointe \\ sont géographiquement très concentrées}

Ces activités ont été sélectionnées, parmi les secteurs de la NAP $600^{5}$, parce qu'elles ont connu des changements dans la technologie du produit ou dans les techniques de production et que ces changements ont pu entraîner des modifications importantes dans la qualification du travail requis et dans la géographie de l'implantation des différentes fonctions ${ }^{6}$. Deux approches, qui se recouvrent partiellement, nous ont aidées dans la sélection :

- le niveau de la fonction «technique», apprécié par la part des effectifs employés dans les études, les recherches, les essais, les contrôles et les laboratoires ${ }^{7}$, quelle que soit la qualification, en 1976 (tableaux 2 et 3). Toutes les activités sélectionnées ont un niveau de fonction technique supérieur au niveau moyen de l'industrie $(6,8 \%)$; certaines consacrent une part énorme des emplois à cette fonction -25 à $40 \%$ des effectifs employés;

- l'importance de la recherche-développement [33], appréciée en termes d'effectifs; ce critère a permis de retenir les activités dont la fonction technique était à peine supérieure à la moyenne, mais qui fournissent un effort intense de recherche : verre plat, pneumatiques, produits photographiques et cinématographiques.

Ces activités ont eu, au cours de la période, des dynamiques de croissance assez variées; il a paru intéressant d'en tenir compte dans la mesure où l'on pouvait imaginer que ces dynamiques influencent les stratégies mises en œuvre (tableau 4).

On peut considérer que, pour le plus grand nombre des activités étudiées, au moins $60 \%$ de l'emploi est concentré dans moins de $10 \%$ des agglomérations de plus de 10000 habitants. Encore faut-il avoir bien présent à l'esprit le poids considérable de l'agglomération parisienne (qui souvent concentre au moins $40 \%$ de l'emploi) et celui des autres grandes agglomérations (plus de 200000 habitants, en particulier). Les petites villes, celles qui comptent moins de 50000 habitants, sont très peu concernées par ces activités. Cette concentration des activités de pointe dans les niveaux supérieurs de la hiérar-
2. Les activités de pointe sélectionnées

\begin{tabular}{|c|c|}
\hline ACTIVITÉS SÉLECTIONNÉES & $\begin{array}{c}\text { SALARIÉS } \\
\text { EMPLOYÉS DANS LA } \\
\text { FONCTION TECHNIQUE } \\
\text { EN } 1976(\%)\end{array}$ \\
\hline A très haute fonction technique & \\
\hline $\begin{array}{l}\text { Construction d'engins et de lanceurs } \\
\text { spatiaux }\end{array}$ & 41,9 \\
\hline $\begin{array}{l}\text { Fabrication de matériel de traitement } \\
\text { de l'information }\end{array}$ & 33,9 \\
\hline $\begin{array}{l}\text { Matériel professionnel électrique et } \\
\text { radio-électrique }\end{array}$ & 28,5 \\
\hline $\begin{array}{l}\text { Matériel de radiologie et d’électroni- } \\
\text { que médicale }\end{array}$ & 27,3 \\
\hline $\begin{array}{l}\text { Construction de cellules d'aéronefs } \\
\text { Fabrication d'équipements spécifi- }\end{array}$ & 26,2 \\
\hline ques pour aéronefs & 25,7 \\
\hline $\begin{array}{l}\text { tisation de processus industriels } \\
\text { Fabrication de propulseurs d'aéronefs }\end{array}$ & 25,7 \\
\hline et d'équipements de propulseurs & 24,2 \\
\hline $\begin{array}{l}\text { A haute fonction technique ou à effort } \\
\text { de recherche élevé }\end{array}$ & \\
\hline $\begin{array}{l}\text { Matériel télégraphique et télépho- } \\
\text { nique }\end{array}$ & 16,2 \\
\hline $\begin{array}{l}\text { Fabrication de caoutchouc synthéti- } \\
\text { que et d'élastomère }\end{array}$ & 15,8 \\
\hline Appareils de contrôle et de régulation & 14,6 \\
\hline $\begin{array}{l}\text { Fabrication de produits de base pour } \\
\text { la pharmacie }\end{array}$ & 14 \\
\hline $\begin{array}{l}\text { Fabrication d'instruments d'optique } \\
\text { et de précision }\end{array}$ & \\
\hline $\begin{array}{l}\text { Fabrication de matières plastiques } \\
\text { Fabrication de produits photographi- }\end{array}$ & 12,2 \\
\hline ques et cinématographiques & 11,8 \\
\hline $\begin{array}{l}\text { Fabrication de transmissions hydrau- } \\
\text { liques et pneumatiques }\end{array}$ & 11 \\
\hline $\begin{array}{l}\text { Fabrication de pneumatiques et de } \\
\text { chambres à air }\end{array}$ & 8,7 \\
\hline $\begin{array}{l}\text { Fabrication et transformation du } \\
\text { verre plat }\end{array}$ & 7,3 \\
\hline
\end{tabular}

Ces activités regroupent en moyenne les deux tiers de leurs salariés dans les unités urbaines de plus de 10000 habitants. Cette proportion atteint 80 à $98 \%$ pour les trois quarts des activités; seules quelques-unes atteignent des proportions plus faibles : produits de base pour la pharmacie, $62 \%$; matières plastiques, $53 \%$; caoutchouc synthétique, $42 \%$; propulseurs d'aéronefs, $60 \%$; instruments d'optique et de précision, $62 \%$.

5. Nomenclature d'activités et de produits, 1973.

6. Cf. l'étude d'E. Decoster et M. TABarie, convention de recherche ministère de l'Urbanisme et du Logement, Université Paris I.

7. Calcul effectué, à partir de l'enquête annuelle d'entreprise et de l'enquête "Structure des emplois », par la STISI. 
3. Caractéristiques de la qualification du travail des activités industrielles de pointe dans les unités urbaines de plus de 10000 habitants

\begin{tabular}{|c|c|c|c|c|c|c|c|c|}
\hline \multirow[t]{2}{*}{ ACTIVITÉS DE POINTE } & \multicolumn{2}{|c|}{$\begin{array}{c}\text { TAUX } \\
\text { DE FÉmINITÉ! } \\
(\%)\end{array}$} & \multicolumn{2}{|c|}{$\begin{array}{c}\text { EMPLOIS DE } \\
\text { PRODUCTION } \\
(\text { En \% du total })\end{array}$} & \multicolumn{2}{|c|}{$\begin{array}{c}\text { TAUX } \\
\text { DE TECHNICITÉ } \\
\text { DES EMPLOIS } \\
\text { DE PRODUCTION }{ }^{2} \\
(\%)\end{array}$} & \multicolumn{2}{|c|}{$\begin{array}{l}\text { QUALIFICATION } \\
\text { OUVRIĖRE }^{3}\end{array}$} \\
\hline & 1975 & 1980 & 1975 & 1980 & 1975 & 1980 & 1975 & 1980 \\
\hline Verre plat & 15,5 & 15 & 75 & 76 & 11 & 10 & 2,4 & 2,5 \\
\hline Produits de base pour la pharmacie & 22,4 & 29 & 75 & 75 & 20 & 33,5 & 2 & 8,2 \\
\hline Matières plastiques & 21 & 13,3 & 62 & 74 & 29 & 20 & 2,5 & 4,5 \\
\hline Caoutchouc synthétique & 10 & 23,5 & 74 & 76 & 17 & 15 & 2,6 & 1,2 \\
\hline $\begin{array}{l}\text { Produts pnotograpniques \& cinematographi- } \\
\text { ques }\end{array}$ & 33,6 & 28 & 48 & 49 & 27 & 24 & 0,7 & 2,1 \\
\hline Transmissions hydrauliques et pneumatiques & 17,5 & 19 & 75 & 75 & 21 & 23 & 1,1 & 1,8 \\
\hline Matériel traitement de l'information & 23,6 & 23,3 & 48 & 46 & 61 & 68 & 1,8 & 3,5 \\
\hline $\begin{array}{l}\text { Equipements d'automatisation de processus in- } \\
\text { dustriels }\end{array}$ & 27 & 21,3 & 67 & 69 & 36 & 48 & 16 & 38 \\
\hline Matériel télégraphique et téléphonique & 48 & 47 & 84 & 81 & $\begin{array}{l}36 \\
23\end{array}$ & $\begin{array}{l}48 \\
27\end{array}$ & $\begin{array}{l}1,0 \\
0,7\end{array}$ & $\begin{array}{l}3,8 \\
1\end{array}$ \\
\hline Radiologie et électronique médicale & 22 & 25,5 & 74 & 70 & 36 & 48 & 2,7 & 2,8 \\
\hline Appareils de contrôle et régulation & 43 & 43 & 76 & 74 & 28 & 30 & 0,8 & 1 \\
\hline $\begin{array}{l}\text { Matériel professionnel électrique et radio- } \\
\text { électrique }\end{array}$ & 34,6 & 31,5 & 77 & 76,5 & 40 & 49 & 1 & 2 \\
\hline Construction de cellules d'aéronefs & 9 & 8 & 82 & 82 & 41 & 40 & 11,3 & 15,7 \\
\hline Propulseurs et équipements de propulseurs & 10,6 & 11 & 83 & 83 & 36 & 38 & 6,1 & 6,8 \\
\hline Equipement spécifique pour aéronefs & 24 & 21 & 82 & 81 & 35 & 37 & 3,3 & 4,2 \\
\hline Engins et lanceurs spatiaux & 15,5 & 16,5 & 72 & 73 & 66 & 74 & 13,2 . & 20 \\
\hline Instruments d’optique de précision & 40 & 30 & 75 & 71 & 21 & 32 & 0,8 & 2,8 \\
\hline Pneumatiques et chambres à air & 15 & 14 & 86 & 86 & 11 & 14 & 0,4 & 0,6 \\
\hline
\end{tabular}

1. Taux de féminité : proportion des femmes dans l'emploi total.

2. Taux de technicité : proportion des cadres, ingénieurs et techniciens dans l'ensemble des emplois.

3. Qualification ouvrière : rapport entre la proportion des ouvriers qualifiés et celle des ouvriers sans qualification.

chie urbaine se conjugue à une forte concentration régionale qui écarte la distribution géographique de ces activités de pointe de celle, plus traditionnelle, des industries de transformation. En effet, près de $50 \%$ des emplois des activités de pointe sont concentrés dans l'Hle-de-France et la région Provence-Côte d'Azur. Mais, à l'intérieur de cet ensemble, les neuf dixièmes sont localisés en région parisienne. Le reste se trouve plutôt localisé dans les régions peu ou récemment industrialisées; les régions du Nord et de l'Est sont, de ce point de vue, particulièrement défavorisées.

Ces caractéristiques de forte concentration géographique sont encore accentuées si l'on considè.e, non plus la distribution géographique de l'ensemble des emplois, mais celle des différents types d'emploi. Les quatre cinquièmes des emplois de cadres, ingénieurs et techniciens sont concentrés dans les agglomérations de plus de 200000 habitants; les deux tiers des ouvriers travaillent dans des villes de plus de 100000 habitants, et cela correspond en fait aux trois quarts des ouvriers qualifiés. Ces distributions très sélectives reflètent certes les tendances générales de la distribution entre les villes des différents types d'emploi observées à propos de l'ensemble des activités, mais elles en sont une expression tout à fait extrême. D'ailleurs, on retrouve les mêmes tendances dans la distribution interrégionale des types d'emplois. L'inégale concentration géographique des emplois des industries de pointe selon le niveau de qualification reflète assez largement les disparités observées à propos de l'ensemble des salariés, mais elle en exacerbe toutes les dissymétries. 
4. Taux de variation du nombre des salariés des activités de pointe entre 1975 et 1980

\begin{tabular}{|c|c|c|}
\hline ACTIVITÉS SÉLECTIONNÉES ${ }^{1}$ & A & $\mathrm{B}$ \\
\hline \multicolumn{3}{|l|}{ 1. Activités en forte croissance } \\
\hline $\begin{array}{l}\text { Equipement d'automatisation de processus } \\
\text { industriels }\end{array}$ & 142 & 184 \\
\hline Produits de base pour la pharmacie & 62 & 154 \\
\hline Matières plastiques & 58 & 167 \\
\hline Transmissions hydrauliques et pneumatiques & 57 & 51 \\
\hline \multicolumn{3}{|l|}{ 2. Activités en croissance moyenne } \\
\hline Equipements spécifiques pour aéronefs & 46 & 15 \\
\hline Engins et lanceurs spatiaux & 40 & 47 \\
\hline Matériel de traitement de l'information & 38 & 8 \\
\hline \multicolumn{3}{|l|}{ 3. Activités en faible croissance } \\
\hline $\begin{array}{l}\text { Produits photographiques et cinématographi- } \\
\text { ques }\end{array}$ & 25 & 66 \\
\hline Caoutchouc synthétique et élastomère & 22 & 69 \\
\hline $\begin{array}{l}\text { Matériel professionnel électrique et radio- } \\
\text { électrique }\end{array}$ & 17 & 10 \\
\hline Pneumatiques & 6 & 5 \\
\hline Propulseurs et éléments de propulseurs & 5 & 13 \\
\hline $\begin{array}{l}\text { Matériel de radiologie et d'électronique médi- } \\
\text { cale }\end{array}$ & 4 & 2 \\
\hline 4. Activités en régression & & \\
\hline Appareils de contrôle et de régulation & -5 & -15 \\
\hline Matériel télégraphique et téléphonique & -8 & -10 \\
\hline Construction de cellules d'aéronefs & -11 & -9 \\
\hline Instruments d'optique et de précision & -20 & -2 \\
\hline Verre plat & -28 & -30 \\
\hline
\end{tabular}

A. Taux de variation de l'emploi total. 1975-1980². (\%)

B. Taux de variation de l'emploi dans les agglomérations de + de 10000 habitants. $1975-1980^{3}$. (\%)

1. N A P 600

2. SOURCE. UNEDIC

3. Source. Enquête "Structure des emplois".

Du fait de la très forte concentration géographique des activités de pointe, encore accentuée pour les emplois les plus qualifiés, les disparités interurbaines du niveau de qualification des emplois que ces activités introduisent sont extrêmes. Le taux de technicité de ces emplois s'élève très régulièrement, et beaucoup plus rapidement que ce que nous avons pu observer pour l'ensemble des activités, avec la taille des agglomérations. La qualification relative des emplois ouvriers augmente elle aussi régulièrement avec la taille des villes, mais les contrastes sont un peu moins accusés.

Les changements intervenus dans les distribu-

8. Expansion due en grande partie aux activités suivantes : produits de base pour la pharmacie, matériel professionnel électrique et radio-électrique, propulseurs d'aéronefs, équipements spécifiques pour aéronefs, engins et lanceurs spatiaux, instruments d'optique de précision. tions des emplois entre 1975 et 1980 , du fait de la «mobilité» interurbaine des industries de pointe, entendue ici au sens de croissance différentielle, n'ont pas été suffisamment importants pour que les très fortes correspondances entre hiérarchie des qualifications et hiérarchie urbaine aient été sérieusement entamées. Quelques retouches cependant ont été apportées qui résultent en fait de stratégies spatiales différentes.

Les activités de pointe (tableau 4) dont le niveau de qualification de l'emploi est très élevé et celles pour lesquelles la période 1975-1980 a été d'amélioration très rapide du taux de technicité des emplois, en rapport généralement avec des changements technologiques importants, ont relativement accentué leur importance en région parisienne. Cette expansion en région parisienne ${ }^{8}$ est en grande partie passée par la création d'emplois ouvriers; elle a contribué au rééquilibrage du réseau, rapprochant un peu de celles des autres villes les structures parisiennes d'emploi de ces activités. En effet, les emplois très qualifiés moins nombreux ont été plutôt créés dans les autres grandes villes du réseau ou dans des villes de taille plus moyenne.

Les activités de pointe moins technicisées (tableau 3), dont les emplois sont en moyenne moins qualifiés, se sont au contraire relativement peu développées dans l'agglomération parisienne. Celles dont les emplois ouvriers sont faiblement qualifiés et dont les emplois de service occupent une proportion de salariés très mineure ont privilégié les agglomérations de moins de 100000 habitants. Celles pour lesquelles niveaux de tertiarisation et de qualification des emplois ouvriers sont plus élevés se sont relativement plus développées dans les unités urbaines de 20000 à 50000 habitants ou dans celles de plus de 200000 habitants.

On doit enfin noter que les agglomérations de moins de 50000 habitants ont été des points de repli privilégié pour les activités de pointe en crise, alors que les activités de pointe en croissance ont été fortement attirées par l'agglomération parisienne.

Le jeu combiné de ces disparités interurbaines de croissance n'a pas sérieusement entamé la très forte correspondance qui existait, en 1975, entre la position hiérarchique des villes et la spécificité de leur emploi pour les activités de pointe. La suprématie de l'agglomération parisienne demeure incontestée dans ce domaine. Si l'écart 
existant entre elle et les autres villes françaises a été un peu réduit, c'est à cause de la concentration relative des emplois ouvriers qualifiés qui s'y est opérée. Ces résultats montrent qu'il n'existe aucune contradiction majeure entre l'évolution récente de la division spatiale du travail des activités de pointe et de l'ensemble des activités, entre les différents niveaux de la hiérarchie urbaine. L'érosion, très relative, de la prééminence parisienne pour le niveau d'encadrement du travail se retrouve, mais plus faible encore, pour l'ensemble des activités. Pour les autres villes, que l'on raisonne sur l'ensemble des activités industrielles ou sur les seules industries de pointe, le rattrapage demeure assez modeste. Une différence peut être notée cependant : dans le premier cas, ce sont plutôt les villes moyennes qui ont profité des redistributions géographiques en cours ; en termes d'effectifs employés, ce sont plutôt les petites villes qui ont été au total les bénéficiaires des sélections opérées par l'agglomération parisienne. En fait, les sélections sont passées au moins autant par l'appartenance régionale des villes que par leur position hiérarchique dans le réseau urbain.

Les schémas interrégionaux de la «mobilité » des industries de pointe sont assez différents de ceux de l'ensemble des industries de transformation. La grande originalité de la croissance différentielle des activités de pointe dans les régions est que cette croissance s'est reportée, prioritairement, sur les réseaux urbains des régions les plus tertiaires et ayant des niveaux de qualification du travail déjà élevés. Cette évolution, qui se fait largement sur la base d'une «mobilité» plus grande des emplois ouvriers, contribue fortement à accentuer les déséquilibres interrégionaux. Les vieilles régions industrielles, en particulier le Nord-Pas-de-Calais, sont fortement pénalisées par ces redistributions différentielles.

En réalité, ces tendances générales résultent de jeux très sélectifs et propres à chacun des types d'activité. Les industries de pointe dont le niveau de qualification du travail, en particulier de technicité, est le plus élevé ou celles dont ce niveau s'est fortement amélioré entre 1975 et 1980 ont largement alimenté la croissance des industries de pointe de la région parisienne surtout et aussi de la région Provence-Côte d'Azur - et à l'intérieur de ces régions les plus grandes villes, nous l'avons déjà noté. Cette croissance a amélioré, en particulier dans ces régions, le niveau de qualification du travail ouvrier et assure le maintien de leur prééminence, en ce qui concerne le taux d'encadrement du travail, initialement très élevé. Les industries de pointe à qualification du travail relativement faible ont eu tendance à accroître leur importance relative dans les villes petites et moyennes des régions où l'activité tertiaire est sousreprésentée et où l'emploi est en moyenne peu qualifié (Auvergne, Franche-Comté, Bourgogne, etc.). Entre ces deux extrêmes, les industries de pointe aux caractéristiques d'emploi plus moyennes ont plutôt privilégié les agglomérations de 20000 à 200000 habitants de régions elles aussi plus moyennes du point de vue de l'importance des fonctions tertiaires et de la technicité du travail, mais où le niveau de qualification des emplois ouvriers est assez élevé (Alsace, Rhône-Alpes, Sud-Ouest).

Enfin, si l'on considère les industries de pointe, non du point de vue de leur structure d'emploi, mais de celui de leur dynamique de croissance, on observe que les replis relatifs qui s'effectuent sur les régions les moins industrialisées concernent bien rarement les activités les plus dynamiques.

Au total, les activités de pointe contribuent d'une manière assez prévisible à la hiérarchisation des espaces régionaux. On ne voit pas se dessiner par elles de nouvelles tendances de restructuration de l'espace national. Certes, les industries de pointe, un peu plus que les autres, contribuent à renforcer l'image du modèle héliotropique. Mais la croissance de l'emploi en dehors de la région parisienne bénéficie d'abord de ce que celle-ci ne sélectionne pas ou ne veut pas conserver. Assurément, la région ProvenceCôte d'Azur a un peu "égratigné » cette suprématie sans partage, et son profil de qualification des emplois des industries de pointe est désormais plus proche de celui de la région parisienne que d'aucune autre région française. Mais les rapports de force entre elles sont restés tellement inégaux qu'il y aurait quelque dérision à voir pour l'instant dans cette région méridionale quelque nouvelle «Californie» française. Le renforcement du modèle "grande ville» de l'emploi, auquel nous venons de montrer que les industries de pointe ont largement contribué, est une tendance du changement récent assurément plus profonde que celle de l'imposition d'un modèle héliotropique de la «mobilité » des activités industrielles. 


\section{Les processus locaux de changement}

On retrouve les grandes tendances du changement des profils de qualification du travail des agglomérations françaises de plus de 10000 habitants dans l'évolution récente de la qualification du travail du bassin d'emploi de Blois : l'enquête menée auprès des établissements de plus de cinquante salariés montre que tertiarisation et qualification des emplois sont effectivement deux tendances locales de l'évolution entre 1975 et 1980 . La tertiarisation repose ici pour l'essentiel sur le développement assez rapide des activités de service - public et parapublic - en particulier jusqu'en 1980 et d'une partie seulement des services marchands. Le processus de qualification du travail, réel et reconnu par tous, résulte d'un jeu complexe, qui ne peut être dissocié de l'accroissement constant, quoique particulièrement modeste à Blois, du nombre des licenciements et de l'augmentation du nombre de personnes à la recherche d'un emploi. Il semble qu'il faille distinguer les processus à l'action à l'intérieur des activités industrielles et ceux qui orientent l'évolution du travail dans les activités tertiaires.

Dans le domaine industriel, les contrastes sont importants selon qu'il s'agit d'établissements à main-d'œuvre majoritairement peu qualifiée et alors souvent féminine - ou d'établissements à niveau de qualification du travail élevé. Dans le premier cas, l'évolution, due en général à une réduction des marchés, se traduit par une réduction des effectifs employés sans modification des structures d'emploi. Dans le second cas, pour les établissements à majorité d'emplois masculins, si la crise affecte l'établissement au point qu'il soit obligé de procéder soit à des licenciements, soit, cas plus fréquent à Blois, à des mises à la retraite anticipées, alors ce sont les ouvriers les moins qualifiés qui sont prioritairement menacés et touchés. Cela se traduit alors par une amélioration de la qualification relative des "profils d'emploi des établissements. Les raisons invoquées de ce choix sont de deux ordres : les ouvriers non qualifiés ont souvent une moindre ancienneté dans l'établissement ; le maintien en place des ouvriers qualifiés est considéré par les chefs d'établissement comme la garantie d'une meilleure aptitude à réagir à une crise économique dont les effets sont de plus en plus prégnants à Blois, à partir de 1980 en particulier. Enfin, la tendance à la qualification peut être accentuée dans de nombreux cas par la mise en place de procédures automatiques qui lèsent prioritairement les ouvriers non qualifiés et suscitent au contraire l'apparition de postes de travail qualifié de contrôle et de maintenance. Ces restructurations de la qualification s'opèrent en différentes circonstances, mais l'occasion dont il est fait état le plus fréquemment est l'allégement inquiétant du carnet de commandes et la recherche d'une réorientation, par la diversification par exemple. Au terme de ces mouvements, la qualification du travail féminin industriel, ouvrier essentiellement, est demeurée très stable entre 1975 et 1982, alors qu'un processus de qualification du travail ouvrier masculin s'est dessiné, sans que par ailleurs la configuration de l'appareil productif ait été modifiée dans ses caractéristiques générales.

C'est dans une conjoncture de croissance du nombre des emplois que se sont opérées à Blois les restructurations de la qualification du travail dans les activités tertiaires. En dépit de la difficulté réelle qu'il y a, sur le terrain, à définir correctement la notion de qualification du travail - des employés surtout -, quelques traits importants des transformations en cours ont pu être mis en évidence.

Un décalage croissant s'instaure, beaucoup plus sensible que dans le secteur industriel, entre le niveau de formation requis pour accéder à un emploi et la qualification effectivement nécessaire - et surtout reconnue dans le poste effectivement occupé. Le volant important et croissant de main-d'œuvre à la recherche d'un emploi, qui est en outre plus jeune et plus diplômé que par le passé, facilite l'accentuation rapide de ce décalage.

L'introduction systématique, dans les plus grands établissements surtout, d'outils de travail de plus en plus sophistiqués (informatique, télématique, bureautique) ne contribue que peu à la modification du niveau de qualification du travail des services, du moins au niveau des emplois d'exécution. Le transfert interne du personnel d'un poste de travail à l'autre se fait le 
plus souvent sans changement de qualification, après une formation accélérée, sous forme de stage pour les employés. Cette introduction a par contre pour résultat de faire appel à de nouvelles catégories de cadres et de techniciens, dont le recrutement est, le plus souvent, extra-régional.

L'élévation générale des niveaux de qualification apparaît nettement dans certaines activités de service du secteur privé. Elle est imputable à une modification de l'activité, qui va dans le sens d'une complexification des services rendus, donc des tâches réalisées, voire à l'apparition de nouvelles activités.

Mais, dans plusieurs activités tertiaires, en particulier dans le secteur public, le niveau de

\section{Bibliographie}

1. Aydalot Ph., Dynamique spatiale et développement inégal, Economica, Paris, 1976.

2. AyDalot Ph., «la Mobilité des activités et de l'emploi », Revue d'économie régionale et urbaine, $\mathrm{n}^{\circ} 3$, octobre 1978.

3. Aydalot Ph., «le Rôle du travail dans les nouvelles stratégies de localisations", Revue d'économie régionale et urbaine, $\mathrm{n}^{\circ} 2,1979$.

4. Aydalot Ph., "Division spatiale du travail et croissance urbaine», Dossiers du Centre Economie, Espace, Environnement, $\mathrm{n}^{\circ} 12$, Université Paris I, 1980.

5. Azouvi A., "Emploi, qualification et croissance dans l'industrie», Economie et Statistique, n' 119, 1980.

6. Boyoux Ph., «Profession ou disparition des qualifications ouvrières", Aspects économiques de l'Ile-deFrance, ${ }^{\circ} 10,1984$.

7. Braibant M., "le Tertiaire insaisissable ?», Economie et Statistique, $\mathrm{n}^{\circ} 146,1982$.

8. Browaeys X., Chatelain P., la France du travail, P U F, Paris, 1984.

9. Centre d'étude et de recherche sur l'emploi et la qualification (CEREQ), Evolution des emplois et des qualifications dans le secteur de l'industrie, des services et des commerces, 1970.

10. CEREQ, la Qualification du travail. De quoi parle-ton?, La Documentation française, Paris, 1978.

11. CÉZARD M., "les Qualifications ouvrières en question », Economie et Statistique, $\mathrm{n}^{\circ} 110,1979$.

12. Clemenceau P., Virville N., "Garçons et filles face à leur insertion professionnelle », Economie et Statistique, $\mathrm{n}^{\circ} 134,1981$.

13. Coeffic N., «le Chômage en octobre 1980 », Economie et Statistique, $\mathrm{n}^{\circ} 133,1981$.

14. Corng H., la Ville, marché de l'emploi, Presses universitaires de Grenoble, Grenoble, 1982.

15. Comité départemental d'action Économique (COD A C), Bassin d'emploi de Blois. Pré-étude : 1. Etude statistique. - 2. La vie économique, 1984

16. Eymard-Duvernay F., «les Secteurs de l'industrie et leurs ouvriers", Economie et Statistique, 1981.

17. FERNANDEZ S., Transformations de l'activité féminine. Facteurs évolutifs, Centre d'étude de l'emploi, dossier $\mathrm{n}^{\circ} 6,1982$.

18. Hueth M., «la Progression de l'activité féminine est-elle irréversible ?», Economie et Statistique, n $145,1982$. qualification des emplois est faible et le taux d'encadrement particulièrement bas. Il est, dans le secteur public de Blois, au moins deux fois inférieur à ce qu'il est dans les établissements bancaires. Or, en dépit de l'élévation continue du niveau de formation à l'embauche, ce taux d'encadrement des emplois de la fonction publique s'est abaissé à Blois entre 1975 et 1982, alors qu'il a eu tendance à fortement augmenter dans les établissements comparables du secteur privé.

L'élévation du taux de chômage et le renforcement relatif, parmi les demandeurs d'emploi, des ouvriers et des employés qualifiés témoignent des limites de ce processus de qualification. Mais en cela la situation du bassin d'emploi de Blois

19. Iribarne A., Virville N., «les Qualifications et leur évolution", in CEREQ, la Qualification. De quoi parle-t-on?, La Documentation française/Commissariat au Plan, Paris, 1978.

20. Lauhle P., «la Montée du chômage féminin", Economie et Statistique, $\mathrm{n}^{\circ} 126,1980$.

21. Lefort R., "la Politique du pire», Options, n* 115 1977.

22. Lipietz A., «la Dimension régionale du développement tertiaire ", Travaux et recherches de prospective, $n^{\circ} 75,1978$.

23. Mallet L., "le Marché local du travail», CNRS, Toulouse, 1980.

24. Marchand C., Revoll J.-P., «Emploi et chômage. Bilan fin 1980 ", Economie et Statistique, n 130 , 1981.

25. Planque B., "les Fonctions de recherche et leur environnement local ", RERU, n 3, 1982.

26 Provendier F., Pumain D., Saint-Julien Th., les Villes dans l'espace social, travaux de recherche de l'Institut de géographie de Reims, 1982.

27. Pumain D., Saint-Julien Th., les Dimensions $d u$ changement urbain, Paris, CNRS, 1978.

28. Pumain D., SAINT-Julien Th., "les Transformations récentes du système urbain français ", l'Espace géographique, $\mathrm{n}^{\circ}$ 3, 1979.

29. SaInT-Julien Th., Croissance industrielle et Système urbain, Economica, Paris, 1982.

30. Saint-Julien Th., la Diffusion spatiale des innovations, collection Reclus-Mode d'emploi, Montpellier, 1985.

31. Saint-Julien Th. et al., la Division interurbaine $d u$ travail en France. La crise et l'évolution de la qualification du travail dans les villes, convention $\mathrm{n}^{\circ} 8131504227501$, ministère de l'Urbanisme et du Logement, Université Paris I, 1984.

32. Sofer C., "Emplois "féminins" et emplois "masculins". Mesure de la ségrégation et évolution de la féminisation des emplois ", Annales de l'INSEE, $n^{\circ} 52,1983$

33. STISI, "les Firmes industrielles et la recherchedéveloppement", Traits fondamentaux du système industriel français, $\mathrm{n}^{\circ}$ 9, 1982.

34. Valeyre A., "Dynamique régionale de l'emploi et division spatiale du travail ", Revue d'économie régionale et urbaine, $\mathrm{n}^{\circ} 3,1982$. 
est encore une bonne image de la situation générale française.

Ainsi peuvent être rapidement résumées les expressions locales des tendances générales de changement. Cette étude à grande échelle permet de cerner les mécanismes suivant lesquels une ville «moyenne » a pu réagir à la crise récente, dans le domaine de l'évolution de la qualification du travail. Elle montre aussi à quel point sont fortes les interdépendances dans un réseau urbain et combien il est difficile de dissocier les évolutions locales observées des processus généraux de transformation, à l'action dans l'ensemble du réseau.

Au cours des cinq années étudiées, l'ampleur et la généralité des processus de la transformation des profils urbains de qualification du travail ont été si importantes qu'elles dominent les changements observés, que l'on raisonne sur l'ensemble des unités urbaines de plus de 10000 habitants ou à plus grande échelle sur une agglomération particulière, que l'on considère l'ensemble des activités ou plus spécialement des activités de pointe, témoins des changements de demain. Tertiarisation et élévation de la qualification ont dominé l'évolution récente des profils de l'emploi salarié des villes françaises. L'analyse à grande échelle réalisée sur le cas particulier de Blois met en évidence l'originalité des mécanismes qui, pour l'activité industrielle d'une part, l'activité tertiaire d'autre part, ont conduit à ces évolutions.

Des vitesses différentes de transformation ont toutefois prolongé ou amorcé quelques redistri- butions géographiques de l'emploi très significatives. Les plus grandes villes ont, dans l'ensemble, continué à renforcer les niveaux de «tertiarisation» et de qualification de leur emploi salarié. L'ensemble des villes de la région parisienne et des agglomérations du sud et surtout du sud-est de la France a bénéficié d'une évolution particulièrement favorable. De ce point de vue, la position de la région parisienne demeure très privilégiée. Pour les plus grandes villes du nord et de l'est de la France, on constate l'apparition d'une relative distorsion entre une structure de qualification plutôt favorable (surtout pour l'emploi industriel) et une évolution récente plutôt plus défavorable que celle des agglomérations de même taille dans d'autres régions. La question de la conservation des potentialités en place y est donc posée et renvoie à celle des moyens à mettre en œuvre pour y répondre. Celle-ci mérite d'autant plus d'attention que nous avons pu montrer la faible attractivité exercée par ces villes septentrionales sur la «mobilité » qualitative récente des activités de pointe.

On peut enfin s'interroger sur la signification à plus long terme des changements observés. Il y a tout lieu de penser que ces changements sont l'expression d'une tendance transitoire qui correspond au stade primaire du processus de diffusion d'une nouvelle révolution technologique. On commence à en saisir l'ampleur, mais nul ne peut avec précision en prévoir la vitesse de propagation. Tendance transitoire certes, mais importante, car elle renforce les déséquilibres anciens en en renouvelant les bases.

Thérèse SaInt-Julien, Denise Pumain

Denise Pumain est maître de conférences à l'Université de Paris I et dégagée de sa charge d'enseignement comme chargée de recherches à l'INED. Thérèse Saint-Julien est maître de conférences à l'Université de Paris I.

Animatrices d'une équipe de recherche sur la dynamique des systèmes urbains, elles ont publié ensemble les Dimensions du changement urbain (Editions du CNRS, 1978) et, séparément : Denise PumaIN, la Dynamique des villes, Economica; Thérèse SaINT-Julien, Croissance industrielles et système urbain, Economica. 